\title{
Experiencia lectora de los alumnos de la licenciatura de ingeniero agrónomo industrial de la UAEMéx
}

\section{Reading experience of the students of the industrial agronomist engineering degree at UAEMex}

\author{
DOI: $10.46932 / s f j d v 3 n 1-060$
}

Received in: Jan 30st, 2021

Accepted in: Feb 1th, 2022

\author{
Dr. en E. Sergio Hilario Díaz \\ Profesor de Tiempo Completo de la Facultad de Ciencias Agrícolas, México \\ E-mail: camaoseh@yahoo.com.mx. \\ Dra. en E. Guadalupe Melchor Díaz \\ Profesora de Tiempo Completo del Centro Universitario UAEM Amecameca, México, \\ E-mail: gpe_md@yahoo.com.mx. \\ M. en E.S. Narciso Campero Garnica \\ Profesor de Tiempo Completo del Centro Universitario UAEM Amecameca, México, \\ E-mail: camperomx53@gmail.com.
}

\begin{abstract}
RESUMEN
Los estudiantes cuando llegan a la universidad enfrentan nuevas formas de pensar, de buscar, adquirir, elaborar y comunicar conocimientos, y por ende de leer textos universitarios. El objetivo de esta investigación es presentar el diagnóstico del uso de estrategias de lectura y el dominio de su aplicación en alumnos de la Licenciatura de Ingeniero Agrónomo Industrial de la UAEMéx. La metodología que se desarrolla es a partir de un estudio descriptivo e interpretativo basado en información y datos de naturaleza cuantitativa y cualitativa, la muestra estuvo conformada por 40 alumnos a quienes se les aplicó una encuesta, el diseño del cuestionario comprende los tres rubros de comprensión lectora (estrategias cognoscitivas, metacognoscitivas y de administración de los recursos) mismas que son aplicadas en tres momentos: antes, durante y después de la lectura. Estas estrategias tienen un uso muy bajo lo que explica la deficiencia de la comprensión lectora.
\end{abstract}

Palabras clave: Lectura de comprensión, estrategias de lectura, experiencia lectora, textos técnicos científicos, aprendizaje de la lectura.

\footnotetext{
ABSTRACT

Students when they arrive at the university face new ways of thinking, searching, acquiring, elaborating and communicating knowledge, and therefore reading university texts. The objective of this research is to present the diagnosis of the use of reading strategies and the mastery of their application in students of the Bachelor of Industrial Agricultural Engineering of the UAEMéx. The methodology that is developed is from a descriptive and interpretative study based on information and data of a quantitative and qualitative nature, the sample was made up of 40 students to whom a survey was applied, the design of the questionnaire includes the three areas of reading comprehension (cognitive, metacognitive and resource management strategies) which are applied at three times: before, during and after the reading.
} 
Keywords: Comprehension reading, reading strategies, reading experience, scientific technical texts, reading learning.

\section{INTRODUCCIÓN}

En los últimos años la lectura se ha convertido en uno de los objetivos esenciales de la educación, ya que el fracaso educativo se ha visto muy relacionado con el escaso dominio de la lengua escrita. Ante esta situación, debemos suponer que el escaso dominio de la lectura se debe a la concepción, que de ella se tiene y la forma de enseñarla, así como a sus nociones de aprendizaje, pues en la educación básica y media superior se ha formado a los alumnos en una mecánica de lectura centrada en la decodificación. Aceptar que esta forma de enseñar a leer es adecuada, implicaría que todos los alumnos de educación básica son lectores, porque, si algo hace este sistema, es leer por obligación un día tras otro. En consecuencia, podemos suponer que desarrollaron el hábito lector, sin embargo no es así, porque desarrollar el hábito de la lectura es una decisión de la voluntad, el hábito de la lectura tiene que ver con las intenciones y tensiones del sujeto frente a un texto, ya que las intenciones determinarán el propósito de su lectura y la tensión genera la necesidad interior de seguir leyendo. Por eso lo que habría que hacer en la escuela es crearles necesidades potenciales, porque la lectura no es una necesidad natural, si no cultural. De ahí que la presente investigación busca conocer el nivel de experiencia en el uso de estrategias de lectura de comprensión de los alumnos del cuarto semestre de la licenciatura en Ingeniero Agrónomo Industrial de la Uaeméx.

\section{METODOLOGÍA}

Al llegar al nivel superior de educación el principal problema al que se enfrentan los alumnos, es el lenguaje. Pues cada ciencia, de las que integran el plan de estudios, tiene un discurso específico. Para representar la realidad que abarca y va transformando en su proceso de desarrollo. Los estudiantes de Ciencias Agrícolas, han demostrado un bajo nivel de comprensión lectora, para apropiarse de manera efectiva del discurso de las diferentes disciplinas de su plan de estudio. Estas observaciones permiten concluir, que el problema lector de los alumnos, no está en el reconocimiento del código, sino que no tienen los conocimientos previos, que el texto requiere, para su desentrañamiento. La incomprensión del sujeto puede deberse a su incapacidad, para identificar lo relevante, para conectar unas ideas con otras, para interpretar ciertas claves que el texto proporciona. Pero, sobre todo, se debe a su impericia para deducir o inferir matices y conclusiones. Para dar respuesta a todo lo anterior, se plantean una serie de preguntas y objetivos de investigación que tiene la intensión de indagar sobre la problemática descrita. 
Pregunta de Investigación: ¿Cuál es el nivel de experiencia en el manejo de estrategias de lectura de comprensión de los alumnos del cuarto semestre de la licenciatura en Ingeniero Agrónomo Industrial de la Facultad de Ciencias Agrícolas de la UAEMéx?

Preguntas particulares: ¿Cuáles son las estrategias de lectura de comprensión que utilizan los alumnos? ¿Cuáles son las estrategias necesarias para que el alumno realice lectura de comprensión de textos de las ciencias naturales?

Objetivo General: Diagnosticar el nivel de experiencia en el uso de estrategias de lectura de comprensión de los alumnos del cuarto semestre de la licenciatura en Ingeniero Agrónomo Industrial de la UAEMéx.

Objetivos particulares: Seleccionar las estrategias de lectura de comprensión, con base en la teoría, para abordar los textos técnicos científicos. Diagnosticar el uso de estrategias de lectura de comprensión y su dominio de aplicación por los alumnos de la licenciatura en Ingeniero Agrónomo Industrial de la UAEMéx. Para diagnosticar las estrategias de lectura de comprensión que emplea el alumno y el grado de dominio que tiene de éstas, se propuso desarrollar una investigación de tipo descriptiva e interpretativa basada en información y datos de naturaleza cuantitativa y cualitativa. Para recoger la información de los alumnos, se efectuó una caracterización del objeto desde la información dada a través de una encuesta, por una muestra intencional de estudiantes de la licenciatura en Ingeniero Agrónomo Industrial. Matriculados en la materia Comunicación profesional. Esta muestra estuvo conformada por 40 alumnos. La encuesta sobre las prácticas de lectura fue extraída del Cuadernillo para el conocimiento y uso de estrategias metacognitivas de lectura para la comprensión de textos científicos (2001) del Instituto Politécnico Nacional. Este cuadernillo fue diseñado con la finalidad de analizar con detalle lo que los alumnos hacen mientras leen un texto técnico científico. El cuestionario está conformado por cuarenta y tres pares de afirmaciones, de las cuales el alumno debe seleccionar una de las dos opciones, considerando la frecuencia con que la utiliza. El diseño del cuestionario considera los tres rubros de comprensión lectora (estrategias cognoscitivas, metacognoscitivas y de administración de los recursos). Para el fin de este estudio, se decidió utilizar únicamente aquellas estrategias empleadas durante el proceso de lectura de comprensión. Para la codificación de las preguntas se realizó un análisis estadístico a través de proporciones. La interpretación de los resultados se realizó a partir de la confrontación de los resultados del análisis con la teoría.

\section{RESULTADOS}

Nivel de comprensión lectora de los alumnos de $4^{\circ}$ semestre de la Licenciatura en Floricultura de la Facultad de Ciencias Agrícolas. (UAEM). 


\begin{tabular}{|l|l|l|l|}
\hline ESTRATEGIAS DE COMPRENSIÓN LECTORA & $\begin{array}{l}\text { PUNTAJE } \\
\text { MÁXIMO }\end{array}$ & $\begin{array}{l}\text { PUNTAJE } \\
\text { OBTENIDO }\end{array}$ & $\%$ \\
\hline LECTURA EN FUNCIÓN DEL PROPÓSITO & 240 & 115 & 48 \\
\hline EXPERIENCIA EN EL USO DE ESTRATEGIAS & 240 & 91 & 38 \\
\hline REVISIÓN PERIÓDICA DE LO LEÍDO & 240 & 163 & 68 \\
\hline IDENTIFICACIÓN DE IDEAS AFINES A LA PRINCIPAL & 240 & 77 & 32 \\
\hline DIFERENTES MANERAS PARA COMPRENDER & 240 & 41 & 17 \\
\hline RELACIÓN DE LAS IDEAS CON EL PROPÓSITO DE LA LECTURA & 240 & 84 & 35 \\
\hline IDENTIFICACIÓN DEL POR QUÉ NO SE ENTIENDE & 240 & 149 & 62 \\
\hline COMPRENSIÓN DE ACUERDO A LA ESTRUCTURA DEL TEXTO & 240 & 110 & 46 \\
\hline BÚSQUEDA DE PALABRAS CLAVE AL INICIO & 240 & 29 & 12 \\
\hline & & & \\
\hline
\end{tabular}

Nivel de dominio de las estrategias de la lectura de comprensión

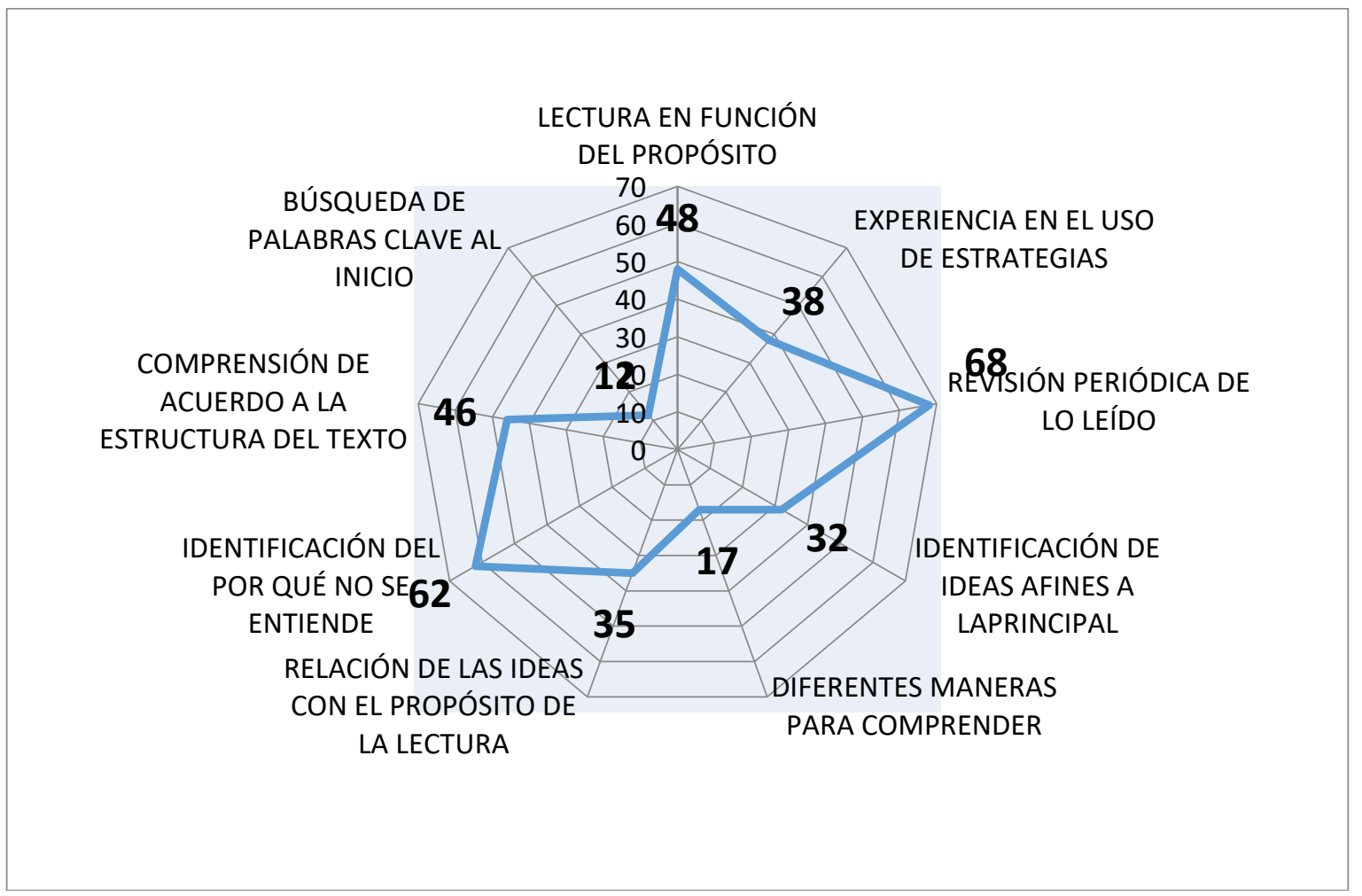

Fuente: Cuestionario de Villegas, 2001

Los resultados de la encuesta ilustran las tendencias en el uso de estrategias de comprensión lectora en los alumnos. En la presentación de los resultados se exponen los aspectos contemplados en la encuesta que se consideran de mayor interés para la reflexión. Con base en los resultados de la encuesta, entre las estrategias que utilizan los estudiantes universitarios al leer textos técnico-científicos, se destaca la "revisión periódica de lo leído", en el que un 68\% afirma que se detiene periódicamente durante su lectura para revisar mentalmente lo que ha leído, siendo la estrategia más empleada por el alumnado. 
Los resultados anteriores muestran que lo más frecuente (68\%) es que los alumnos se detengas periódicamente en su lectura para revisar lo que han leído. Esto es, según Teresita Villegas (2001), una fortaleza; siempre y cuando las pausas sean para identificar las ideas más importantes que permiten construir la comprensión; sin embargo, en comunicación directa con los alumnos, han indicado que las principales razones por las que se detienen son la pérdida de concentración, falta de léxico especializado, la complejidad misma de los textos, pero, principalmente, porque no buscan comprender el texto, sino memorizarlo mecánicamente, para lo que necesitan asegurarse de que han retenido toda la información.

Continuando con los resultados de la encuesta, el $62 \%$ de los alumnos se da cuenta por qué no entiende lo que lee. De acuerdo con Teresita Villegas (2001), esta es una fortaleza en los alumnos. Dentro de las principales interferencias señaladas como factores que les obstaculizan entender lo que leen, sobresale la desviación de la atención, inducida por el uso de celular, las lecturas no significativas, falta de léxico especializado, las preocupaciones personales y sociales, y la necesidad de estar acompañado o conversando con frecuencia.

\section{CONCLUSIONES}

En la información obtenida se pudo observar que en todos los datos que se recolectaron y analizaron se manifiesta un uso de las diferentes estrategias útiles durante la lectura de comprensión. Esta experiencia en el uso efectivo de las estrategias de lectura se encuentra en diferentes niveles de dominio. Destacándose "revisión periódica de lo leído" y "la identificación de por qué no se entiende. El papel de las instituciones, como la Facultad de Ciencias Agrícolas, dedicada a la educación agrícola superior, es formar a sus alumnos como lectores competentes. La dificultad no radica en el conocimiento del código, "sino en que no cuenta con los conocimientos previos que el texto requiere para su desentrañamiento". Esto se percibió claramente durante el análisis de los resultados, al detectar su incapacidad para identificar sistemáticamente lo relevante del texto, para conectar las ideas principales entre sí. Pero, sobre todo, su impericia para deducir, inferir o producir una idea global del texto. Lo anterior lo prepara para la comprensión y la interpretación, "pues sin comprensión no hay nada, ni aprendizaje significativo ni cognitivo, ni de ninguna otra especie". Respaldar la formación académica exclusivamente en la lectura de los apuntes y en la explicación de los profesores (actividad cotidiana en los alumnos). Estar sujeto a construir un conocimiento a partir de información que, en muchos casos, resulta imparcial e imprecisa. Debido a la espontaneidad con que se elabora, induciendo a que el alumno emplee la memorización mecánica como su principal estrategia de aprendizaje. En lugar de desarrollar la capacidad y las estrategias necesarias para aprender a partir de la construcción del conocimiento. 


\section{REFERENCIAS}

Andere Martínez, Eduardo (2010), El poder de PISA Listas de ranking y mejores prácticas en educación internacional, Nuevo Pensamiento, México, 255 pp.

Argudin, Yolanda; María Luna (1994), Aprender a pensar leyendo bien, Plaza y Valdes Editores, México, 259 pp.

Burón, Javier (s/n), Enseñar a aprender: introducción a la metacognición, Ediciones Mensajero, Bilbao, 157 pp.

Calderón Ibañez, Arlenys; Quijano Pañuela, Jorge; "Características de comprensión lectora en estudiantes universitarios” en Revista Estudios Socio-Jurídicos, vol. 12, núm. 1, enero-junio, 2010. pp. 337-364.

Carlino, Paula (2010), Escribir, leer y aprender en la universidad Una introducción a la alfabetización académica, FCE, México, 200 pp.

Castro Daza, Diana Patricia; Rosa María Niño Gutierrez (2013), Tendencias sobre las prácticas de lectura y escritura en la universidad colombiana: el punto de vista de los estudiantes

Fajardo Hoyos, Adriana; Hernández Jaramillo, Janeth; González Sierra, Ángela, “Acceso léxico y comprensión lectora: un estudio con jóvenes universitarios" en REDIE. Revista Electrónica de Investigación Educativa, vol. 14, núm. 2, 2012, pp. 25-33.

Fontes Martínez, Carlos Fabián (2013), “Evaluación de las políticas de tecnologías de la información y la comunicación en la educación pública federal" en Estudios sobre reformas educativas: visiones en contrapunto, Universidad Pedagógica Nacional, México, pp. 179-202.

Goldin, Daniel (Coord.) (2006), Encuesta nacional de lectura Informes y evaluaciones, CONACULTA / UNAM, México, 286 pp.

Gordillo Alfonso, Adrián (2013), Las bases textuales y los géneros discursivos

Maqueo, María Ana (2009), Lengua, aprendizaje y enseñanza

Mayor, Juan ( ), Estrategias metacognitivas: aprender a aprender y aprender a pensar

Moreno, Víctor (2011), ¿Cómo formar lectores competentes? Guía práctica: reflexiones y propuestas, Pamiela, México, 191 pp.

Moreno Suárez, Irma Patricia; Gómez López, Luis Felipe, "El proceso de comprensión lectora en alumnos de tercero de secundaria", en Revista Mexicana de Investigación Educativa, vol. 18, núm. 56, enero-marzo, 2013, pp. 113-139.

Parodi, Giovanni (Coord.) (2010), Saber leer, Aguilar, México, 223 pp.

Pérez, Héctor ( ), Comprensión y producción de textos

Rincón B., Gloria; John Saúl Gil (2013) Lectura y escritura académica en la Universidad del Valle, Programa Editorial Universidad del Valle, Colombia, $281 \mathrm{pp}$.

Sandoval, Aída; Franchi, Lissette (2007), Metacomprensión en estudiantes de ingeniería

Simone, Raffaele (2001), La tercera fase Formas de saber que estamos perdiendo, Taurus, México, 155 pp.

Villegas López, Teresita del N.J. (2001), Cuadernillo para el conocimiento y uso de estrategias metacognitivas de lectura para la comprensión de textos científicos, Instituto Politécnico Nacional, México, 123 pp. 


\section{NOTAS BIBLIOGRÁFICAS}

Sergio Hilario Díaz (Ejutla de Crespo, Oaxaca) Doctor en Educación por el Centro de Estudios Superiores en Educación (CESE). Es Maestro en Administración de Organizaciones por la Universidad Nacional Autónoma de México e Ingeniero Agrónomo Fitotecnísta, por la Universidad Autónoma del Estado de México. Actualmente, se desempeña como profesor de tiempo completo de la Facultad de Ciencias Agrícolas, UAEM. Coordina el Departamento de Evaluación profesional y Responsable del Programa de Fomento a la Lectura.

Guadalupe Melchor Díaz (Chalco, Estado de México) Doctora en Educación por el Centro de Estudios Superiores en Educación (CESE). Es Maestra en Administración de Organizaciones por la Universidad Nacional Autónoma de México y Licenciada en Relaciones Internacionales, también por la UNAM. Actualmente, se desempeña como profesora de tiempo completo de la Licenciatura en Nutrición en el Centro Universitario UAEM Amecameca. Responsable del Programa de Fomento a la Lectura del Centro Universitario UAEM Amecameca.

Narciso Campero Garnica (México, D.F.) Maestro en Enseñanza Superior por la UNAM e Ingeniero Químico por la misma Institución; Profesor de Educación Primaria por la Benemérita Escuela Nacional de Maestros, Ex Director del C.U. UAEM Amecameca, Ex Coordinador del Programa Tutorial, Ex Coordinador de la Licenciatura Nutrición en el mismo espacio y actualmente Profesor de Tiempo Completo del C.U. UAEM Amecameca. 